\title{
Habilidades cognitivas e desempenho nos testes de ordenação temporal em idosos
}

\section{Cognitive abilities and performance in the temporal ordering tests for elderly people}

\author{
Maysa Bastos Rabelo' (D), Marcia da Silva Lopes $^{2}$ (D), Ana Paula Corona² (D), Jozélio Freire de Carvalho ${ }^{1}$, \\ Roberto Paulo Correia de Araújo ${ }^{3}$
}

\section{RESUMO}

Objetivo: Investigar a influência dos domínios cognitivos no desempenho do teste padrão de frequência e teste padrão de duração em idosos. Métodos: estudo seccional, desenvolvido em indivíduos com idade entre 60 e 79 anos. Realizou-se entrevista, avaliação cognitiva por meio do Montreal Cognitive Assessment, avaliação audiológica periférica (audiometria tonal e vocal) e central (teste padrão de frequência e teste padrão de duração). Resultados: Participaram do estudo 58 mulheres com média de idade de 66 anos e 2 meses e 28 homens, com média de idade de 68 anos e 3 meses. Verificou-se que as habilidades visuoespacial, de atenção, concentração e memória de trabalho apresentaram correlação com os testes temporais no sexo feminino e que a habilidade de linguagem apresentou correlação com o teste padrão de frequência. Já entre os homens, houve tendência à significância quanto à capacidade visuoespacial. Ademais, as mulheres apresentaram melhor desempenho na habilidade de memória. Conclusão: Os aspectos cognitivos podem influenciar nos testes de ordenação temporal em indivíduos idosos, sobretudo do sexo feminino.

Palavras-chave: Audição; Transtornos da percepção auditiva; Envelhecimento; Idoso; Cognição

\begin{abstract}
Purpose: To investigate the influence of cognitive domains on the performance of the pitch pattern sequence and duration pattern sequence tests among elderly people. Methods: Cross-study realized among individuals aged between 60 to 79 years. We performed an interview, cognitive evaluation through Montreal Cognitive Assessment, peripheral audiologic evaluation (tonal and vocal audiometry) and central (pitch pattern sequence and duration pattern sequence). Results: The study involved 58 women with average age of 66.2, and $28 \mathrm{men}$ at an age average of 68.3. On the one hand, we observed that the visual-spatial abilities, attention, concentration and working memory present correlation with temporal tests in females and that language ability correlates with the standard frequency test. Among men, there was a tendency to significance in terms of visual-spatial abilities. Furthermore, women had better performance in memory ability. Conclusion: Cognitive aspects may influence the temporal ordering tests among elderly individuals, especially females.
\end{abstract}

Keywords: Hearing; Auditory perception disorders; Aging; Elderly; Cognition

\footnotetext{
Trabalho realizado no Instituto de Ciências da Saúde, Universidade Federal da Bahia - UFBA - Salvador (BA), Brasil.

${ }^{1}$ Programa de Pós-graduação Processos Interativos dos Órgãos e Sistemas, Instituto de Ciências da Saúde, Universidade Federal da Bahia - UFBA - Salvador (BA), Brasil.

${ }^{2}$ Departamento de Fonoaudiologia, Instituto de Ciências da Saúde, Universidade Federal da Bahia - UFBA - Salvador (BA), Brasil.

${ }^{3}$ Departamento de Bioquímica Oral, Instituto de Ciências da Saúde, Universidade Federal da Bahia - UFBA - Salvador (BA), Brasil.

Conflito de interesses: Não.

Contribuição dos autores: MBR participou da idealização do estudo, coleta, análise e interpretação dos dados e redação do artigo; MSL e APC participaram da análise, interpretação dos dados e redação do artigo; JFC e RPCA realizaram a revisão intelectual do artigo.

Financiamento: Coordenação de Aperfeiçoamento de Pessoal de Nível Superior (CAPES), Brasil. Processo no 1620297.

Autor correspondente: Maysa Bastos Rabelo. E-mail: maysa_bastos@yahoo.com.br

Recebido: Novembro 20, 2019; Aceito: Maio 04, 2020
} 


\section{INTRODUÇÃO}

A avaliação comportamental do processamento auditivo temporal é baseada na realização de testes que investigam as habilidades de resolução, mascaramento, integração e ordenação. Esta última pode ser analisada mediante testes que avaliam o reconhecimento do padrão temporal de tons puros, como o teste padrão de frequência (TPF) e o teste padrão de duração (TPD) ${ }^{(1)}$.

O processamento temporal permite ao indivíduo identificar variações sutis que ocorrem na informação acústica, no decorrer do tempo, que contribuem para os processos de análise e decodificação do estímulo acústico, apresentando impacto sobre a inteligibilidade de fala ${ }^{(2)}$. Ademais, em decorrência do envelhecimento, as dificuldades relacionadas à percepção de fala implicam a queixa de dificuldade de compreensão, principalmente em ambientes ruidosos, que não apresenta relação com a alteração auditiva periférica ${ }^{(3)}$.

Estudos demonstraram relação entre o declínio da memória de trabalho relacionado à idade e dificuldades no processamento auditivo, sobretudo nos aspectos temporais ${ }^{(4)}$. Adicionalmente, a modalidade de resposta verbal nos testes temporais envolve processos cognitivos refinados, que dependem da integridade de ambos os hemisférios e do corpo caloso ${ }^{(5)}$.

Assim, ao interpretar os achados nos testes temporais, sem considerar o desempenho em tarefas que requerem um aporte de habilidades cognitivas, pode-se culminar na superestimativa de dificuldades de idosos em relação ao processamento auditivo.

Diante do exposto, o objetivo do presente estudo foi investigar quais os domínios cognitivos apresentam influência no desempenho do teste padrão de frequência e teste padrão de duração, em idosos.

\section{MÉTODOS}

O presente estudo do tipo seccional foi aprovado pelo Comitê de Ética em Pesquisa do Instituto de Ciências da Saúde da Universidade Federal da Bahia (UFBA), protocolo de número 2.268.734, e todos os participantes assinaram o Termo de Consentimento Livre e Esclarecido.

Obteve-se uma amostra por conveniência de indivíduos que frequentavam centros sociais e de saúde de referência para idosos, na cidade de Salvador/BA. Os critérios de seleção foram: idade maior ou igual a 60 anos, não apresentar relato de traumatismo cranioencefálico, de acidente vascular encefálico e diagnóstico de distúrbio psiquiátrico grave ou doenças neurodegenerativas. Excluíram-se indivíduos com perda auditiva condutiva, mista ou sensorioneural, com grau a partir de moderadamente severo em uma das orelhas e com quadro cognitivo alterado, a partir da versão em português do questionário Montreal Cognitive Assessment (MoCA) ${ }^{(6)}$.

As avaliações foram realizadas no Centro Docente Assistencial de Fonoaudiologia (CEDAF) da UFBA. Foram investigados os aspectos cognitivos, mediante o MoCA, instrumento de rastreio cognitivo em que são avaliados oito domínios. A pontuação máxima no teste é 30 e valores inferiores a 26 indicam comprometimento cognitivo ${ }^{(6)}$.

Para realização das avaliações audiológicas periféricas (audiometria tonal liminar e vocal) e centrais (testes de ordenação temporal), utilizou-se o audiômetro AC40, fones supra-aurais TDH 39 e os indivíduos permaneceram em cabina acústica.
Os testes de processamento auditivo central (PAC) foram realizados de forma binaural, com um tablet marca Samsung, acoplado ao audiômetro, a 50 decibéis nível de sensação, a partir do limiar de recepção de fala. Utilizaram-se três sequências para treinamento e 30 sequências de avaliação. Aqueles que, após três tentativas, não conseguiram discriminar as sequências de treinamento, retornaram outro dia para refazer a avaliação.

No TPF, apresentaram-se sequências de tons puros, diferindo quanto à frequência, sendo alta $(1122 \mathrm{~Hz})$, ou baixa $(880 \mathrm{~Hz})$. Para o TPD, as sequências foram de três tons de $1000 \mathrm{~Hz}$, que diferiram quanto à duração, sendo curtos (250 ms), ou longos $(500 \mathrm{~ms})^{(1)}$. Em ambos os testes, foi solicitada a nomeação do estímulo.

Realizou-se a análise descritiva das variáveis sexo, idade e escolaridade, bem como do desempenho nos testes temporais e dos domínios cognitivos. O MoCA foi analisado considerando-se o escore total e a pontuação obtida nos domínios cognitivos, a saber: função executiva; capacidade visuoespacial; memória, atenção, concentração e memória de trabalho; linguagem e orientação (temporal e espacial).

Investigou-se a associação entre a pontuação total e cada domínio cognitivo, de acordo com o sexo, por meio do teste de Mann-Whitney. O desempenho nos testes de ordenação temporal foi correlacionado aos domínios cognitivos, utilizando-se o coeficiente de correlação de Spearman. O nível de significância estabelecido para este estudo foi de $5 \%$.

\section{RESULTADOS}

Compareceram para avaliação 131 indivíduos, porém, 39 foram excluídos, sendo o motivo mais frequente alteração no aspecto cognitivo $(n=27)$, seguido do diagnóstico de perda auditiva condutiva ou mista $(n=7)$ e não compreensão dos testes de ordenação temporal, mesmo com pontuação igual ou superior a 29 no MoCA $(n=5)$. Para as análises, 6 mulheres não foram consideradas, pois não foram incluídos homens na mesma faixa etária.

Desta forma, foram incluídos 86 indivíduos, a maioria do gênero feminino $(67,4 \%)$, com média de idade de 66 anos e 2 meses. Já no gênero masculino, a média de idade foi 68 anos e 3 meses. Considerando a heterogeneidade da amostra em relação ao gênero, as análises foram realizadas segundo esta variável. Em relação à escolaridade, a maioria das mulheres (39,7\%) e dos homens (42,9\%) tinha segundo grau incompleto ou completo; $22,4 \%$ das mulheres e $28,6 \%$ dos homens tinham nível superior incompleto ou completo e os demais, até primeiro grau de escolaridade.

Ao comparar o desempenho nos diferentes domínios do MoCA, observou-se que apenas a memória apresentou diferença entre os gêneros, sendo que as mulheres obtiveram melhor desempenho (Tabela 1).

A correlação entre o desempenho nos testes temporais e os domínios do MoCA demonstrou que, entre as mulheres, as habilidades visuoespacial, de atenção, concentração e memória de trabalho apresentaram correlação positiva moderada e significativa em ambos os testes de ordenação temporal. Além disso, também entre as mulheres, o domínio linguagem e a pontuação total no MoCA apresentaram correlação positiva moderada e significativa apenas com o TPF. Já entre os homens, identificou-se correlação positiva moderada, com tendência à 
Tabela 1. Mediana e associação dos domínios cognitivos avaliados através do Montreal Cognitive Assessment entre os gêneros

\begin{tabular}{lccc}
\hline \multicolumn{1}{c}{ Variável } & Masculino & Feminino & valor de $\mathbf{p}$ \\
\hline Função executiva & 3,0 & 3,0 & 0,716 \\
Capacidade visuoespacial & 4,0 & 4,0 & 0,319 \\
Atenção, concentração e memória de trabalho & 6,0 & 6,0 & 0,124 \\
Linguagem & 6,0 & 6,0 & 0,073 \\
Memória & 3,0 & 4,0 & $0,019^{*}$ \\
Orientação & - & - & - \\
Pontuação total & 27,5 & 28,0 & 0,832 \\
\hline
\end{tabular}

Teste não paramétrico (Teste $U$ de Mann-Whitney de amostras independentes); ${ }^{*}$ Valor de $p \leq 0,05$

Tabela 2. Correlação entre os domínios cognitivos e os testes de ordenação temporal

\begin{tabular}{|c|c|c|c|c|}
\hline \multirow{2}{*}{ Variável } & \multicolumn{2}{|c|}{ Masculino } & \multicolumn{2}{|c|}{ Feminino } \\
\hline & TPF & TPD & TPF & TPD \\
\hline \multicolumn{5}{|l|}{ Função executiva } \\
\hline Valor de $p$ & 0,66 & 0,48 & 0,590 & 0,626 \\
\hline \multicolumn{5}{|l|}{ Capacidade visuoespacial } \\
\hline Coeficiente de correlação & 0,349 & 0,369 & 0,322 & 0,328 \\
\hline Coeficiente de correlação & 0,062 & 0,168 & 0,428 & 0,449 \\
\hline Valor de $p$ & 0,754 & 0,394 & $0,001^{*}$ & $0,000^{*}$ \\
\hline \multicolumn{5}{|l|}{ Linguagem } \\
\hline Coeficiente de correlação & 0,121 & 0,080 & 0,326 & 0,217 \\
\hline Valor de $p$ & 0,538 & 0,684 & $0,013^{*}$ & 0,102 \\
\hline Coeficiente de correlação & - & - & - & - \\
\hline Valor de $p$ & - & - & - & - \\
\hline \multicolumn{5}{|l|}{ Pontuação total } \\
\hline Coeficiente de correlação & 0,098 & 0.086 & 0,393 & 0,251 \\
\hline Valor de $p$ & 0,619 & 0,663 & $0,002^{*}$ & 0,057 \\
\hline
\end{tabular}

*Valor de $p \leq 0,05$; Correlações não paramétricas

Legenda: TPF = Teste Padrão de Frequência; TPD = Teste Padrão de Duração

significância, para a habilidade visuoespacial em ambos os testes de processamento temporal (Tabela 2).

Os domínios cognitivos que apresentaram correlação com os testes de ordenação temporal são apresentados nas Figuras 1 e 2.

\section{DISCUSSÃo}

Os resultados revelaram que o desempenho de mulheres nos testes de ordenação temporal apresentou correlação com as capacidades visuoespacial, de atenção, concentração, memória de trabalho e linguagem. Para os homens, apenas a capacidade visuoespacial revelou correlação com os testes temporais. Ademais, a memória apresentou diferença entre os gêneros, com maior desempenho para as mulheres.

As alterações cognitivas são descritas na literatura como fatores que influenciam no baixo desempenho em testes de $\mathrm{PAC}^{(3,5)}$. Logo, a variabilidade no desempenho da ordenação temporal em idosos pode ser atribuída, também, a aspectos não auditivos, relacionados ao envelhecimento, como a atenção, memória e competência linguística ${ }^{(7)}$.
A diferença encontrada no presente estudo sobre o comportamento cognitivo entre os gêneros pode ser atribuída a diferenças anatômicas e/ou funcionais, sobretudo no córtex temporal, na área de Broca e no corpo caloso. Este último apresenta maior concentração de fibras nervosas nas mulheres, proporcionando melhor conexão inter-hemisférica ${ }^{(8)}$. Entretanto, autores relataram que tais diferenças são atribuídas a estratégias de utilização dos recursos cognitivos e não das habilidades, logo, apresentariam maior relação com o funcionamento cerebral do que com a anatomia ${ }^{(9)}$. Assim, acredita-se que, em virtude da maior facilidade em integrar diversas habilidades, as mulheres se utilizam mais de recursos cognitivos para responder aos testes, do que os homens.

A capacidade visuoespacial envolve a ativação, a retenção e a manipulação de representações mentais, logo, depende do processamento da informação e da memória de trabalho, considerando os traços de armazenamento, verbal ou visual ${ }^{(10)}$. Uma vez que existe relação da capacidade visuoespacial com a memória de trabalho, acredita-se que a interdependência desses domínios contribui para o melhor desempenho nos testes temporais, entre aqueles com melhor desenvolvimento dessa função cognitiva. 

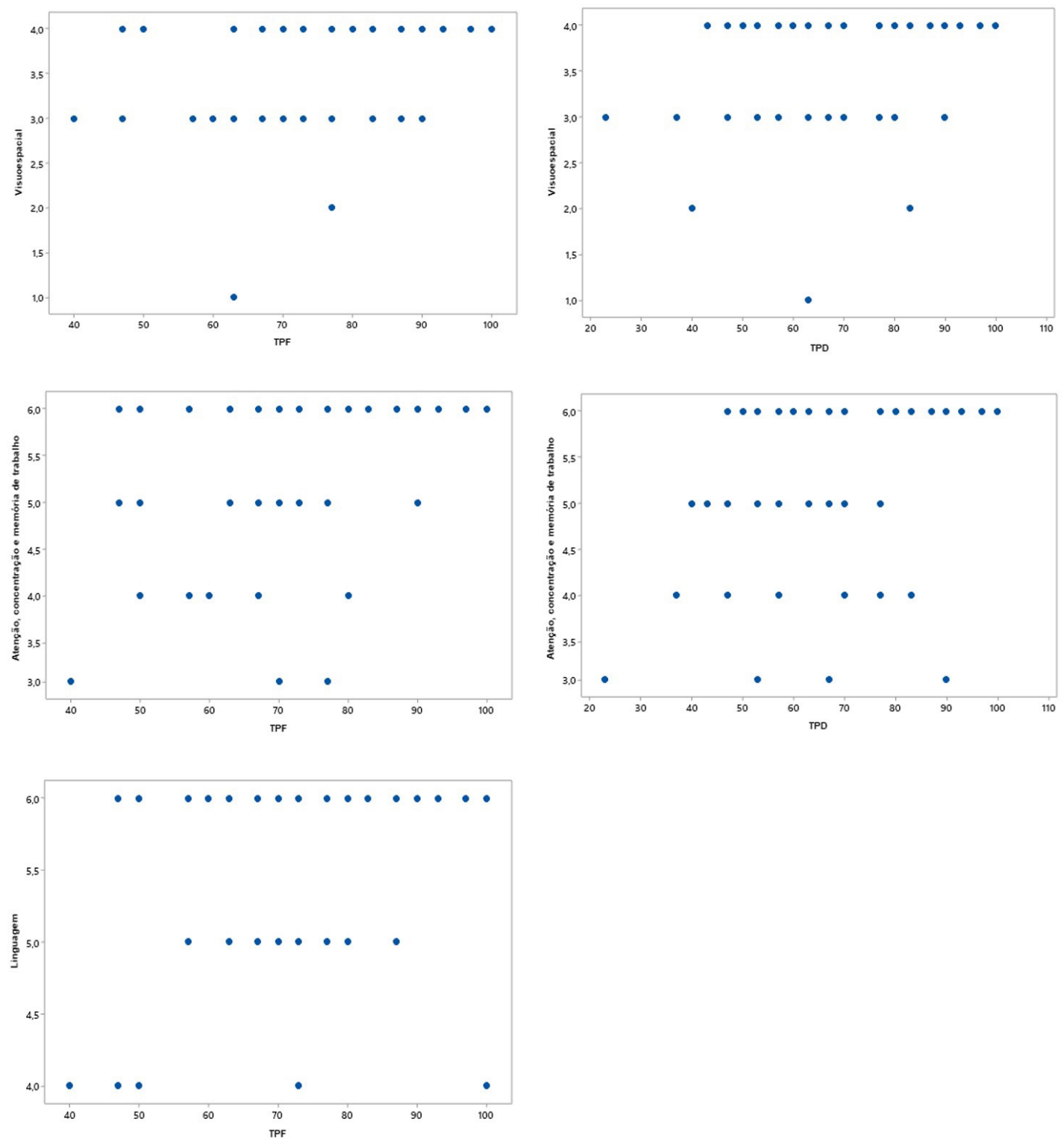

Figura 1. Diagrama de dispersão para correlação entre os domínios cognitivos e o desempenho nos testes de ordenação temporal no sexo feminino

Legenda: TPF = Teste Padrão de Frequência; TPD = Teste Padrão de Duração
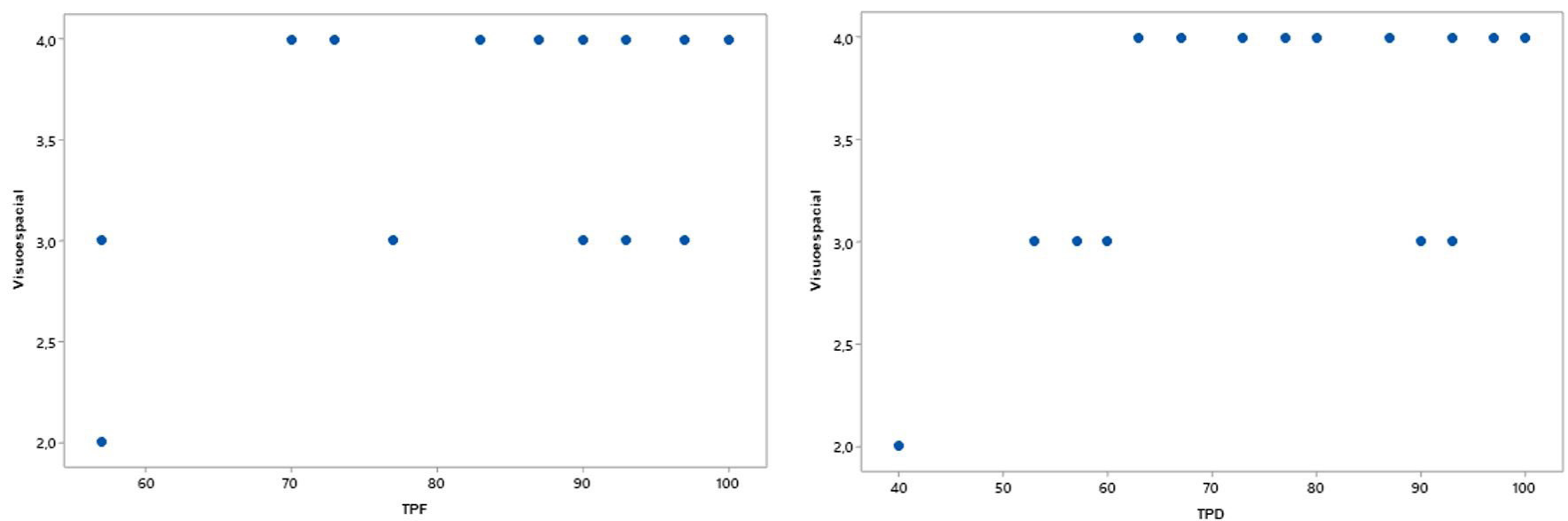

Figura 2. Diagrama de dispersão para correlação entre o domínio cognitivo visuoespacial e o desempenho nos testes de ordenação temporal no sexo masculino

Legenda: TPF = Teste Padrão de Frequência; TPD = Teste Padrão de Duração 
A correlação entre as habilidades de atenção, concentração e memória de trabalho com os testes temporais, observada no estudo atual, é confirmada por outros autores, uma vez que os testes temporais não são exclusivos da modalidade auditiva, pois o estímulo deve ser temporariamente armazenado antes de ser verbalizado, o que envolve tais habilidades, além da competência linguística ${ }^{(11)}$.

A resposta verbal exige a integridade dos hemisférios cerebrais e do corpo caloso para percepção e nomeação ${ }^{(4)}$. Assim, a linguagem é fundamental nos testes temporais, conforme identificado no estudo atual e, quanto melhor essa habilidade, melhor o desempenho nos testes.

Sugere-se que a estimulação diária de funções corticais favorece a sincronia entre os hemisférios cerebrais e a integração com as habilidades auditivas ${ }^{(12)}$. Ademais, quanto maior o processo de organização cerebral, maior a reserva cognitiva, propiciando o aumento da atividade e densidade sináptica, implicando melhora na percepção, memória e raciocínio e consequente retardo no aparecimento do declínio cognitivo ${ }^{(13)}$.

No presente estudo, verificou-se diferença entre os gêneros para habilidade de memória, com melhor desempenho entre as mulheres, concordando com investigação prévia ${ }^{(14)}$. Entretanto, essa investigação merece cautela, uma vez que a memória exigida para realização dos testes temporais é a memória de trabalho, relacionada ao processamento de informações, que se refere à aquisição e ao armazenamento recente, diferente da memória de longo prazo $^{(15)}$.

A principal limitação deste estudo foi com relação ao número reduzido de indivíduos do gênero masculino, o que pode ter contribuído para que as variáveis analisadas não tenham sido estatisticamente significativas nesse grupo. Contudo, vale ressaltar que este é um fenômeno esperado na população idosa, em virtude do maior cuidado com as questões de saúde entre as mulheres e, consequente aumento da expectativa de vida. Assim, investigações adicionais devem ser realizadas com um grupo maior de homens.

A partir deste estudo, verificou-se que a cognição é fundamental para o desempenho das habilidades auditivas, logo, deve ser avaliada previamente à aplicação de testes de PAC, visando nortear a investigação diagnóstica de idosos suscetíveis ao declínio cognitivo, em decorrência da idade.

\section{CONCLUSÃO}

Os achados do presente estudo revelaram que, em idosos, o desempenho nos testes de ordenação temporal é influenciado por habilidades visuoespaciais. Indicaram, também, que, nas mulheres, este desempenho também apresenta associação com a atenção, concentração, memória de trabalho e linguagem. Desta forma, os resultados apontaram que, para desempenhar as tarefas requeridas pelos testes de ordenação temporal, homens e mulheres se utilizam do aporte de habilidades cognitivas diferentes.

\section{REFERÊNCIAS}

1. Musiek FE. Frequency (pitch) and duration patterns tests. J Am Acad Audiol. 1994;5(4):265-8. PMid:7949300.
2. Ghitza O. Linking speech perception and neurophysiology: speech decoding guided by cascaded oscillators locked to the input rhythm. Front Psychol. 2011;2:130. http://dx.doi.org/10.3389/fpsyg.2011.00130. PMid:21743809.

3. Roque L, Karawani H, Gordon-Salant S, Anderson S. Effects of age, cognition, and neural encoding on the perception of temporal speech cues. Front Neurosci. 2019;13:749. http://dx.doi.org/10.3389/ fnins.2019.00749. PMid:31379494.

4. Gates GA, Anderson ML, Feeney MP, McCurry SM, Larson EB. Central auditory dysfunction in older persons with memory impairment or Alzheimer dementia. Arch Otolaryngol Head Neck Surg. 2008;134(7):771-7. http://dx.doi.org/10.1001/archotol.134.7.771. PMid: 18645130 .

5. Deperon TM, Silva IMC, Caldas FF, Mendes BCA, Novaes BCAC. Processamento temporal auditivo em idosos. Distúrb Comun. 2016;28(3):530-8.

6. Nasreddine ZS, Phillips NA, Bédirian V, Charbonneau S, Whitehead V, Collin I, et al. The Montreal Cognitive Assessment, MoCA: a brief screening tool for Mild Cognitive Impairment. J Am Geriatr Soc. 2005 Abr;53(4):695-9. http://dx.doi.org/10.1111/j.1532-5415.2005.53221.x. PMid:15817019.

7. Hennig TR, Costa MJ, Rossi AG, Moraes AB. Efeitos da reabilitação auditiva na habilidade de ordenação temporal em idosos usuários de próteses auditivas. J Soc Bras Fonoaudiol. 2012;24(1):2633. http://dx.doi.org/10.1590/S2179-64912012000100006. PMid:22460369.

8. Guz W, Pazdan D, Stachyra S, Świętoń F, Poręba P, Bednarz M, et al. Analysis of corpus callosum size depending on age and sex. Folia Morphol. 2018;78(1):24-32. PMid:30009363.

9. Ritchie SJ, Cox SR, Shen X, Lombardo MV, Reus LM, Alloza C, et al. Sex differences in the adult human brain: evidencefrom 5216 UK biobank participants. Cereb Cortex. 2018;28(8):2959-75. http://dx.doi. org/10.1093/cercor/bhy109. PMid:29771288.

10. Galera C, Garcia RB, Vasques R. Componentes funcionais da memória Visuoespacial. Estud Av. 2013;27(77):29-43. http://dx.doi.org/10.1590/ S0103-40142013000100004.

11. Mukari SZ, Umat C, Othman NI. Effects of age and working memory capacity on pitch pattern sequence test and dichotic listening. Audiol Neurotol. 2010;15(5):303-10. http://dx.doi.org/10.1159/000283007. PMid:20150728.

12. Silveira KMM, Borges ACLC, Pereira LD. Memória, interação e integração em adultos e idosos de diferentes níveis ocupacionais, avaliados pelos testes da avaliação simplificada e teste dicótico de dígitos. Distúrb Comun. 2004;16(3):313-22.

13. Chiu NT, Lee BF, Hsiao S, Pai MC. Educational level influences regional cerebral blood flow in patients with Alzheimer's disease. J Nucl Med. 2004;45(11):1860-3. PMid:15534055.

14. Souza VL, Borges MF, Vitória CMS, Chiappetta ALML. Profile of cognitive abilities in normal aging. Rev CEFAC. 2010;12(2):186-92. http://dx.doi.org/10.1590/S1516-18462009005000056.

15. Appaiah Konganda S, Sharma M, Monaghan JJ, Keidser G, Valderrama Valenzuela JT, Newall J, et al. Auditory, cognitive, and linguistic processing skills in individuals with hearing loss. J Acoust Soc Am. 2018;143(3):1865. http://dx.doi.org/10.1121/1.5036118. 